\title{
How Biofilm Growth Affects Candida-Host Interactions
}

\author{
Emily F. Eix and Jeniel E. Nett* \\ Departments of Medicine and Medical Microbiology and Immunology, University of Wisconsin-Madison, Madison, WI, \\ United States
}

Candida spp. proliferate as surface-associated biofilms in a variety of clinical niches. These biofilms can be extremely difficult to eradicate in healthcare settings. Cells within biofilm communities grow as aggregates and produce a protective extracellular matrix, properties that impact the ability of the host to respond to infection. Cells that disperse from biofilms display a phenotype of enhanced pathogenicity. In this review, we highlight host-biofilm interactions for Candida, focusing on how biofilm formation influences innate immune responses.

Keywords: Candida, biofilm, host, neutrophil, macrophage, matrix, immunity, dispersion

\section{INTRODUCTION}

\section{OPEN ACCESS}

Edited by:

Juliana Campos Junqueira, São Paulo State University, Brazil

Reviewed by:

Cheshta Sharma,

The University of Texas Health Science Center at San Antonio, United States

Renátó Kovács,

University of Debrecen, Hungary

*Correspondence:

Jeniel E. Nett

jenett@medicine.wisc.edu

Specialty section: This article was submitted to Fungi and Their Interactions, a section of the journal

Frontiers in Microbiology

Received: 12 March 2020 Accepted: 03 June 2020 Published: 25 June 2020

Citation: Eix EF and Nett JE (2020) How Biofilm Growth Affects Candida-Host

Interactions.

Front. Microbiol. 11:1437. doi: 10.3389/fmicb.2020.01437
Candida spp. are the primary cause of nosocomial fungal infections and recently rose to the leading pathogen group causing nosocomial bloodstream infections (Magill et al., 2014). Candida spp. exhibit the propensity to proliferate as adherent biofilms (Magill et al., 2014; Nobile and Johnson, 2015). These aggregated communities exhibit resistance to antifungals as well as host immune responses, making them extremely difficult to eradicate (Chandra et al., 2001a; Donlan, 2001b; Douglas, 2003). In the hospital setting, Candida spp. form biofilms on artificial medical devices, such as vascular catheters, which can lead to bloodstream infection and disseminated disease with associated mortality of approximately 30\% (Donlan, 2001a; Kojic and Darouiche, 2004; Wisplinghoff et al., 2004; Kumamoto and Vinces, 2005; Pfaller and Diekema, 2007; Tumbarello et al., 2007). It is estimated that nearly $80 \%$ of patients with invasive candidiasis have implanted medical devices (Andes et al., 2012). The ability of Candida spp. to persist as a biofilm on these devices poses a serious issue for treatment of Candida infections, as device removal is often the only option (Pfaller and Diekema, 2007; Andes et al., 2012). However, even with catheter removal, mortality rates remain high in the setting of invasive candidiasis (Andes et al., 2012). The observation that removal of catheters decreases the risk of persistent candidemia and rate of mortality suggests that biofilm formation plays a major role in the pathogenesis of invasive candidiasis (Andes et al., 2012; Ala-Houhala and Anttila, 2020).

Candida albicans, the most prevalent Candida spp., has served as a model organism for study of biofilm formation (Hawser and Douglas, 1994; Chandra et al., 2001b; Ramage et al., 2001; Uppuluri et al., 2010). However, biofilm formation is not unique to C. albicans, as many other clinically relevant Candida spp., including C. glabrata, C. tropicalis, C. parapsilosis, and the emerging pathogen C. auris, also form biofilms (Kuhn et al., 2002; Lewis et al., 2002; Jain et al., 2007; Bizerra et al., 2008; Sherry et al., 2017). While biofilms formed by different Candida spp. may vary in morphology and density, the structures uniformly contain a polymeric extracellular matrix that encases and protects the fungal cells. The components of the extracellular matrix differ from those found in the Candida cell wall, and these moieties are proposed to modulate host recognition by concealing the cell wall components that typically interact with the immune system 
(Johnson et al., 2016; Zawrotniak et al., 2017; Hoyer et al., 2018). In addition, cells dispersed from biofilms exhibit characteristics distinct from cells growing under non-biofilm conditions (Sellam et al., 2009; Uppuluri et al., 2010). In this review, we highlight key host interactions with Candida biofilms, describing how the host responds differently to Candida during biofilm and non-biofilm growth (Figure 1).

\section{IMPACT OF BIOFILM FORMATION ON NEUTROPHIL RESPONSES}

Neutrophils serve as primary innate immune responders to Candida and are critical for controlling invasive infection (FidelJr., 2002). The susceptibility of neutropenic patients to severe fungal infections, including candidiasis, highlights the importance of these leukocytes (EdwardsJr., Lehrer et al., 1978; Erwig and Gow, 2016). However, when growing as a biofilm, Candida resists damage and killing by neutrophils (Katragkou et al., 2010, 2011a,b; Xie et al., 2012; Johnson et al., 2016; Kernien et al., 2017). Compared to the neutrophil response to planktonic C. albicans, neutrophils exhibit an up to 5-fold lower activity against biofilms formed by C. albicans (Katragkou et al., 2010, 2011b; Xie et al., 2012; Johnson et al., 2016). This process appears to involve induction of an inhibitory pathway, as neutrophils exposed to C. albicans resist activation by potent stimuli, such as phorbol ester. Additionally, priming of neutrophils with pro-inflammatory cytokines, including interferon $-\gamma$ and granulocyte colony-stimulating factor, does not restore neutrophil killing of biofilms (Katragkou et al., 2011b). This inhibitory process appears independent of filamentation, as C. albicans biofilms of various architectures, including those comprised primarily of yeast morphotypes, similarly impair neutrophil function. Consistent with this, biofilms formed by C. glabrata and C. parapsilosis, which lack true hyphae, also resist neutrophil attack (Katragkou et al., 2011a; Johnson et al., 2017).

Neutrophils respond to pathogens via a variety of effector mechanisms, including phagocytosis, production of reactive oxygen species (ROS), and the formation of neutrophil extracellular traps (NETs), which are web-like structures containing DNA, histones, and antimicrobial proteins (Brinkmann et al., 2004). While neutrophils are capable of phagocytosing Candida yeast cells, the elongated hyphal cells cannot be completely engulfed. Instead, in response to C. albicans hyphae and other large or aggregated pathogens, neutrophils release NETs (Urban et al., 2006; Branzk et al., 2014). This process of NET formation would arguably be an effective neutrophil response against biofilm, considering their large size and the inability of the structures to be completely phagocytosed. However, NETs are not produced in response to C. albicans biofilms (Johnson et al., 2016; Kernien et al., 2017). C. albicans biofilms do not trigger neutrophils to generate ROS, a signaling pathway that governs many forms of NET formation (Brinkmann et al., 2004; Xie et al., 2012; Johnson et al., 2016). These impaired responses have been attributed, in part, to the presence of an extracellular matrix encasing the cells (Johnson et al., 2016).

\section{MONOCYTE AND MACROPHAGE RESPONSES TO BIOFILM}

The formation of biofilm influences a variety of mononuclear innate immune cell responses, including migration, phagocytosis, and cytokine production (Chandra et al., 2007; Katragkou et al., 2010; Alonso et al., 2017; Simitsopoulou et al., 2018; Arce Miranda et al., 2019). In addition, the presence of these and other cells can impact biofilm formation by Candida. For example, when incubated with $C$. albicans biofilm, peripheral blood mononuclear cells fail to engage in phagocytosis (Chandra et al., 2007). However, the presence of these cells promotes the formation of a thicker, hyphal-rich biofilm, which has been linked to a biofilm-enhancing soluble factor produced by the mononuclear cells during co-culture with biofilm (Chandra et al., 2007).

In addition to avoiding phagocytosis, mononuclear cells exposed to biofilms also display an altered cytokine profile, when compared to those interacting with planktonic C. albicans (Chandra et al., 2007). Observed differences for biofilm exposure include increases in both pro-inflammatory cytokines (IL-1 $\beta$ and MCP-1), as well as the anti-inflammatory cytokine, IL-10 (Chandra et al., 2007). Additional studies utilizing a human monocytic cell line (THP-1) have also shown differing cytokine responses to biofilm and planktonic $C$. albicans, including lower TNF- $\alpha$ production upon biofilm exposure (Katragkou et al., 2010). Modulation of cytokine production by biofilm likely influences immunity, but little is known about this process. However, it appears that cytokine responses to Candida biofilm may vary among species (Simitsopoulou et al., 2018).

Investigations using murine macrophage cell lines are beginning to shed light on the impact of Candida biofilm formation on macrophage interactions (Alonso et al., 2017; Arce Miranda et al., 2019). During the initiation of biofilm formation, macrophages are capable of phagocytosing C. albicans (Arce Miranda et al., 2019). However, as biofilms mature, macrophages do not exhibit activity against them, and may even enhance biofilm production (Arce Miranda et al., 2019). This pattern of impaired activity against mature biofilms is similar to that observed for both human mononuclear cells and neutrophils (Chandra et al., 2007; Katragkou et al., 2010, 2011a,b; Xie et al., 2012; Johnson et al., 2016; Kernien et al., 2017). Like the neutrophil response to $C$. albicans biofilms, macrophagebiofilm interactions also involve diminished ROS production (Xie et al., 2012; Johnson et al., 2016; Arce Miranda et al., 2019). Another response impaired by C. albicans biofilms is macrophage migration. Murine macrophages move at rates approximately 2fold lower in response to biofilm when compared to incubation with planktonic C. albicans (Alonso et al., 2017).

\section{ROLE OF Candida BIOFILM MATRIX IN IMMUNITY}

The development of Candida biofilm begins with adherence to a substrate, which is followed by proliferation and the assembly of an extracellular matrix, a hallmark characteristic of mature 


\section{Host-Candida Biofilm Interactions}

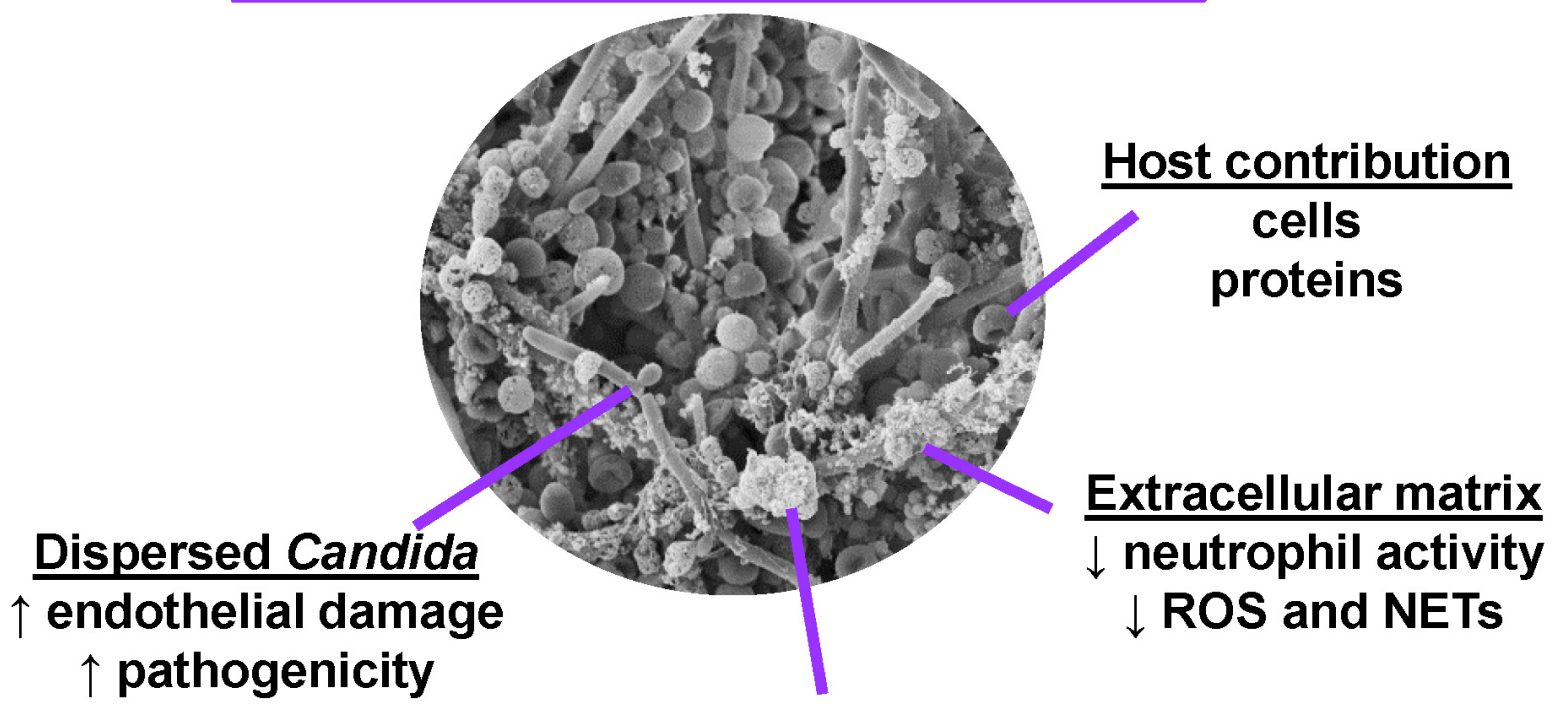

\section{Altered immunity cytokine profiles \\ $\downarrow$ macrophage activity}

FIGURE 1 | Overview of host interactions with Candida biofilms. Scanning electron micrograph reveals C. albicans (SC5314) growing as a biofilm on the luminal surface of a rat vascular catheter. Biofilm formation influences host interactions, immunity, and pathogenesis.

biofilm formation (Chandra et al., 2001a; Uppuluri et al., 2010; Wall et al., 2019). This extracellular matrix encases the cells and presents unique structures which conceal the cell wall components that are typically encountered by innate immune cells (Chaffin et al., 1998; Hawser et al., 1998). Many studies have demonstrated altered immune cell interactions with Candida biofilms (Chandra et al., 2007; Katragkou et al., 2011a; Kernien et al., 2017; Xie et al., 2012; Johnson et al., 2016, 2017; Alonso et al., 2017; Arce Miranda et al., 2019). For neutrophils, this phenotype appears to require an intact extracellular matrix, as disruption of biofilm matrix can restore neutrophil activity, including their ability to produce NETs and damage biofilms (Katragkou et al., 2010; Johnson et al., 2016). It is likely that extracellular matrix contributes to other aspects of immune modulation by biofilms as well.

Analysis of C. albicans biofilms demonstrates that extracellular matrix contains a mixture of biopolymers, including proteins (55\%), carbohydrates (25\%), lipids (15\%), and nucleic acids (5\%) (Zarnowski et al., 2014). Many of these components differ from those found in the cell wall. For example, the biofilm extracellular matrix of $C$. albicans contains an abundant high molecular weight $\alpha$-1,2-branched $\alpha-1,6$ mannan, which assembles with linear $\beta-1,6$ glucan to form a mannan-glucan complex that is not found in the cell wall (Chaffin et al., 1998; Zarnowski et al., 2014; Mitchell et al., 2015). This complex has been linked to the capacity of $C$. albicans biofilms to inhibit neutrophil function, as C. albicans biofilms with genetic disruption of this pathway activate neutrophils (Johnson et al., 2016). Neutrophils then generate ROS and produce NETs in response to these mutants lacking extracellular matrix, ultimately resulting in fungal damage (Johnson et al., 2016). The fungal components involved in triggering this response are not certain. The finding that neutrophils are also activated by biofilms following treatment with echinocandin drugs, which unmask $\beta-1,3$ glucan, suggests a role for this polysaccharide (Katragkou et al., 2010; Hoyer et al., 2018; Simitsopoulou et al., 2018).

The production of matrix mannan-glucan complex is conserved across Candida species, including C. albicans, C. tropicalis, C. parapsilosis, C. glabrata, and C. auris (Dominguez et al., 2018, 2019). It is anticipated that similar mechanisms of neutrophil evasion may occur upon encounter with biofilms formed by these species as well. However, species-specific immune responses have been observed (Simitsopoulou et al., 2018). Additional study is needed to delineate the neutrophilbiofilm interactions for these and other emerging species.

\section{HOST RESPONSE TO DISPERSED BIOFILMS}

Throughout biofilm development, cells detach from biofilms, allowing Candida to disseminate to the bloodstream and cause 
invasive disease (Uppuluri et al., 2010). Various environmental responses, including carbon source and $\mathrm{pH}$, trigger this regulated process for C. albicans (Sellam et al., 2009; Uppuluri et al., 2010). During dispersion, yeast-like cells bud from the upper biofilm layer of hyphae and are release as elongated cells (Uppuluri et al., 2010). Although the cells resemble yeast, the newly dispersed cells display enhanced pathogenicity traits, including heightened capacities for filamentation, adhesion, and biofilm formation (Uppuluri et al., 2010). Even more striking, the dispersed cells exhibit enhanced virulence in a murine model of invasive candidiasis and exert more damage to endothelial cells (Uppuluri et al., 2010). The transcriptional profile of dispersed cells broadly differs from both biofilm and planktonic C. albicans, consistent with their unique phenotype (Sellam et al., 2009; Uppuluri et al., 2018). These cells likely play a major role in the pathogenesis of vascular catheter and other device-associated infections that result in disseminated disease. In this setting, environmental cues can trigger the detachment of yeast-form cells with a heightened propensity to disseminate though the bloodstream, adhere to endothelial cells, and damage tissues (Uppuluri et al., 2010).

\section{INSIGHT INTO HOST RESPONSES TO BIOFILM THROUGH ANIMAL MODELS}

Candida spp. interact extensively with the host during infection, and animal models are ideal for examining this interface in vivo. Invasive candidiasis frequently involves biofilm proliferation on indwelling vascular catheters, which can lead to catheterassociated bloodstream infection and disseminated disease (Kojic and Darouiche, 2004; Andes et al., 2012). Models in mice, rats, and rabbits mimicking vascular catheter-associated infection have shed light on biofilm-host interactions (Andes et al., 2004; Schinabeck et al., 2004; Lazzell et al., 2009). These models reveal differences between in vitro and in vivo biofilms, including the formation of a thicker biofilm matrix in vivo. Interestingly, in vivo biofilm models reveal that a striking number $(>95 \%)$ of host-derived proteins incorporate into the extracellular matrix, indicating a major host contribution to C. albicans biofilms (Nett et al., 2015). Host proteins depositing in the biofilm matrix include matricellular proteins, and proteins indicating the presence of erythrocytes and leukocytes. Imaging of catheters similarly shows the incorporation of many host cells, including erythrocytes and neutrophils (Andes et al., 2004). These findings suggest that neutrophils recruit to C. albicans biofilms in vivo, but lack significant antibiofilm activity.

Urinary catheter biofilm models have been developed in mice and rats, allowing the study of catheter-associated candiduria (Wang and Fries, 2011; Nett et al., 2014; Capote-Bonato et al., 2018). As seen with the vascular catheter models, on urinary catheters, C. albicans also forms thick biofilms with dense extracellular matrix (Wang and Fries, 2011; Nett et al., 2015). Biofilms in this environment also incorporate numerous host cells and proteins, which contribute to the extracellular matrix (Nett et al., 2015). C. tropicalis similarly forms biofilms on urinary catheter segments in mice (Capote-Bonato et al., 2018). It appears that host response to both C. albicans and C. tropicalis biofilms involves a degree of neutrophilic infiltration (Nett et al., 2015; Capote-Bonato et al., 2018). However, the biofilms persist despite this response.

In addition to the vascular and urinary placement of catheters, models have employed subcutaneous implantation of catheters or other devices in mice and rats to elucidate host interactions with Candida biofilms (Ricicova et al., 2010; Nieminen et al., 2014; Kucharikova et al., 2015). These models primarily involve the insertion of preformed biofilms that continue to propagate in vivo. Both $C$. albicans and C. glabrata proliferate as biofilms in this setting (Ricicova et al., 2010; Nieminen et al., 2014; Kucharikova et al., 2015). The subcutaneous C. albicans biofilms induce an infiltration of inflammatory cells consisting predominantly of neutrophils and macrophages (Nieminen et al., 2014). Consistent with this, tissue sections adjacent to subcutaneous biofilms show an inflammatory profile with an increased abundance of inflammatory mediators, including matrix metalloproteinases and myeloperoxidase (Nieminen et al., 2014). Similar to other models of C. albicans biofilm formation, the biofilms withstand this defense.

Oral biofilms represent one of the most common niches for Candida biofilm formation (Douglas, 2002). Rats have predominantly been utilized to study host-biofilm interactions, particularly for the analysis of dental devices and the associated denture stomatitis (Nett et al., 2010; Lee et al., 2011; Johnson et al., 2012; Tobouti et al., 2016; Sultan et al., 2019; Yano et al., 2019). In these models, Candida spp. adhere to the artificial devices, proliferating as a biofilm (Nett et al., 2010; Chen et al., 2011; Johnson et al., 2012; Tobouti et al., 2016; Sultan et al., 2019). Similar to other sites of infection, host materials become intertwined in the extracellular matrix of these C. albicans biofilms, with integration of salivary proteins and immune cells into the matrix (Nett et al., 2015). The mucosal response involves the recruitment of inflammatory cells to the palate mucosa and epithelial changes consistent with the histopathology of dental stomatitis seen clinically (Nett et al., 2010; Johnson et al., 2012; Tobouti et al., 2016; Sultan et al., 2019).

While the clinical relevance of Candida biofilm in the pathogenesis of vulvovaginal candidiasis is not well-understood, murine vaginitis models reveal that C. albicans forms biofilm on the vaginal mucosa (Harriott et al., 2010). These mucosal biofilms are characterized by the presence of an extracellular matrix surrounding yeast and hyphal cells, typical of in vitro C. albicans biofilms and biofilms formed at other sites of infection (Harriott et al., 2010). Clinically, vulvovaginal candidiasis is associated with a robust neutrophil response leading to acute inflammation. A similar acute inflammatory response is recapitulated in a murine model of $C$. albicans infection, which appears to be mediated by the release of S100 alarmins from epithelial cells (Yano et al., 2010, 2012). Interestingly, this likely represents a species-specific host interaction, as C. glabrata does not form biofilm or elicit a strong inflammatory response in this model (Nash et al., 2016). Further studies are required to fully elucidate the complex host response to Candida biofilms during vaginal infection. 


\section{HOST RESPONSES TO Candida IN MIXED-SPECIES BIOFILMS}

Many studies have focused on the proliferation of Candida in a single-species biofilm, but Candida spp. also form polymicrobial biofilms in a variety of niches, including the oropharynx and skin. In these mixed-species biofilms, the immune response to one species may influence immunity to another organism. For example, Staphylococcus aureus has been shown to preferentially adhere to C. albicans hyphae and form mixed-species biofilms (Peters et al., 2010; Zago et al., 2015; de Carvalho Dias et al., 2017). In this setting C. albicans promotes the phagocytosis of Staphylococcus aureus, which can be carried by phagocytes from the oral cavity to the lymphatic system and cause invasive, disseminated disease in murine model of candidiasis (Allison et al., 2019). In addition, formation of a mixed biofilm triggers differential production of soluble factors and proteins that are anticipated to modulate immunity and enhance the virulence of both species (Peters et al., 2010; de Carvalho Dias et al., 2017).

Chronic wounds frequently become colonized by polymicrobial biofilms, and Candida spp. are increasingly recognized as major contributors to these infections (Kalan et al., 2016; Kalan and Grice, 2018). Not only are Candida spp. among the most frequently isolated fungal pathogens from diabetic foot ulcers, the presence of fungi in these wounds correlates with longer healing times (Kalan et al., 2016). In vitro wound biofilm models recapitulate the chronic wound environment and shed light on interactions among common colonizers, including C. albicans, Pseudomonas aeruginosa, and Staphylococcus aureus in this context (Townsend et al., 2016, 2017). In a three-dimensional wound biofilm model, combined antibiotic and antifungal treatments are most effective in eliminating polymicrobial biofilms, emphasizing the importance of considering fungal presence in chronic wounds (Townsend et al., 2017). While much of the host response to these chronic wound biofilms has not yet been elucidated, this represents an important topic for future studies.

In addition to forming polymicrobial biofilms with bacteria, C.albicans also establishes mixed-species biofilms with other Candida (Pathirana et al., 2019; Tati et al., 2016; Vipulanandan et al., 2018). One common clinical example of this is oropharyngeal candidiasis, which often involves multiple Candida species (Redding, 2001). In a mouse model of oropharyngeal candidiasis, colonization by C. glabrata requires the presence of C. albicans (Tati et al., 2016). Co-culture of the organisms lead to upregulation of C. glabrata cell surface proteins that allow for adhesion to C. albicans hyphae (Tati et al., 2016). Additional examples of species involved in mixedspecies biofilms include C. dubliniensis and C. tropicalis, both

\section{REFERENCES}

Ala-Houhala, M., and Anttila, V. J. (2020). Persistent versus non-persistent candidaemia in adult patients in 2007-2016: a retrospective cohort study. Mycoses 63, 617-624. doi: 10.1111/myc.13085 of which appear to adhere to C. albicans and exhibit a growth benefit (Pathirana et al., 2019). When co-cultured together, these species form biofilms that achieve higher surface coverage. The influence of these altered biofilm structures on host responses remains unclear. However, the host interface for mixed biofilms may be quite distinct from that observed for either species alone.

\section{CONCLUSIONS AND FUTURE DIRECTIONS}

Candidiasis frequently involves the formation of surfaceassociated biofilms. These structures have a multifaceted interaction with the host. Compared to cells grown in freefloating conditions, Candida biofilms exhibit resistance to phagocytosis by neutrophils, monocytes, and macrophages. In addition, biofilm formation alters mononuclear cell cytokine profiles, broadly influencing immunity. Biofilms modulate immunity throughout various developmental stages. During mature biofilm formation, extracellular matrix contributes to resistance to host defenses. As fungal cells disperse, a more virulent phenotype results in enhanced pathogenesis.

Further understanding of the impact of biofilm formation on host immunity will be of interest. Many biofilm studies have explored immune cell interactions ex vivo. However, biofilm composition is highly impacted by in vivo conditions, and little is known about how the host contribution to biofilm may alter immune recognition. In addition, studies are just beginning to shed light on the complexity of immunity to mixed biofilms. Furthermore, it will fascinating to see how biofilm formation by emerging species, such as $C$. auris, influences host responses, as C. albicans has primarily been utilized as a model organism.

\section{AUTHOR CONTRIBUTIONS}

$\mathrm{EE}$ and JN wrote the manuscript. Both authors contributed to the article and approved the submitted version.

\section{FUNDING}

JN was supported by the National Institutes of Health (R01 AI145939), the Burroughs Welcome Fund (1012299), and the Doris Duke Charitable Foundation (112580130). EE was supported by the University of Wisconsin Graduate School, part of the Office of Vice Chancellor for Research and Graduate Education, with funding from the Wisconsin Alumni Research Foundation.

Allison, D. L., Scheres, N., Willems, H. M. E., Bode, C. S., Krom, B. P., and Shirtliff, M. E. (2019). The host immune system facilitates disseminated Staphylococcus aureus disease due to phagocytic attraction to Candida albicans during coinfection: a case of bait and switch. Infect. Immun. 87:e00137-19. 
Alonso, M. F., Gow, N. A. R., Erwig, L. P., and Bain, J. M. (2017). Macrophage migration is impaired within Candida albicans biofilms. J. Fungi (Basel) 3:31. doi: 10.3390/jof3030031

Andes, D., Nett, J., Oschel, P., Albrecht, R., Marchillo, K., and Pitula, A. (2004). Development and characterization of an in vivo central venous catheter Candida albicans biofilm model. Infect. Immun. 72, 6023-6031. doi: 10.1128/ iai.72.10.6023-6031.2004

Andes, D. R., Safdar, N., Baddley, J. W., Playford, G., Reboli, A. C., Rex, J. H., et al. (2012). Impact of treatment strategy on outcomes in patients with candidemia and other forms of invasive candidiasis: a patient-level quantitative review of randomized trials. Clin. Infect. Dis. 54, 1110-1122. doi: 10.1093/cid/cis021

Arce Miranda, J. E., Baronetti, J. L., Sotomayor, C. E., and Paraje, M. G. (2019). Oxidative and nitrosative stress responses during macrophage-Candida albicans biofilm interaction. Med. Mycol. 57, 101-113. doi: 10.1093/mmy/ myx143

Bizerra, F. C., Nakamura, C. V., de Poersch, C., Estivalet Svidzinski, T. I., Borsato Quesada, R. M., Goldenberg, S., et al. (2008). Characteristics of biofilm formation by Candida tropicalis and antifungal resistance. FEMS Yeast Res. 8, 442-450. doi: 10.1111/j.1567-1364.2007.00347.x

Branzk, N., Lubojemska, A., Hardison, S. E., Wang, Q., Gutierrez, M. G., Brown, G. D., et al. (2014). Neutrophils sense microbe size and selectively release neutrophil extracellular traps in response to large pathogens. Nat. Immunol. 15, 1017-1025. doi: 10.1038/ni.2987

Brinkmann, V., Reichard, U., Goosmann, C., Fauler, B., Uhlemann, Y., Weiss, D. S., et al. (2004). Neutrophil extracellular traps kill bacteria. Science 303, 1532-1535. doi: 10.1126/science.1092385

Capote-Bonato, F., Bonato, D. V., Ayer, I. M., Magalhaes, L. F., and Magalhaes, G. M. (2018). Pereira da Camara Barros FF, et al. Murine model for the evaluation of candiduria caused by Candida tropicalis from biofilm. Microb. Pathog. 117, 170-174. doi: 10.1016/j.micpath.2018.02.036

Chaffin, W. L., Lopez-Ribot, J. L., Casanova, M., Gozalbo, D., and Martinez, J. P. (1998). Cell wall and secreted proteins of Candida albicans: identification, function, and expression. Microbiol. Mol. Biol. Rev. 62, 130-180. doi: 10.1128/ mmbr.62.1.130-180.1998

Chandra, J., Kuhn, D. M., Mukherjee, P. K., Hoyer, L. L., McCormick, T., and Ghannoum, M. A. (2001a). Biofilm formation by the fungal pathogen Candida albicans: development, architecture, and drug resistance. J. Bacteriol. 183, 5385-5394.

Chandra, J., McCormick, T. S., Imamura, Y., Mukherjee, P. K., and Ghannoum, M. A. (2007). Interaction of Candida albicans with adherent human peripheral blood mononuclear cells increases C. albicans biofilm formation and results in differential expression of pro- and anti-inflammatory cytokines. Infect. Immun. 75, 2612-2620. doi: 10.1128/iai.01841-06

Chandra, J., Mukherjee, P. K., Leidich, S. D., Faddoul, F. F., Hoyer, L. L., Douglas, L. J., et al. (2001b). Antifungal resistance of candidal biofilms formed on denture acrylic in vitro. J. Dent. Res. 80, 903-908. doi: 10.1177/00220345010800031101

Chen, Y. L., Brand, A., Morrison, E. L., Silao, F. G., Bigol, U. G., Malbas, F. F. Jr., et al. (2011). Calcineurin controls drug tolerance, hyphal growth, and virulence in Candida dubliniensis. Eukaryot. Cell 10, 803-819. doi: 10.1128/ec.00310-10

de Carvalho Dias, K., Barbugli, P. A., de Patto, F., Lordello, V. B., de Aquino Penteado, L., Medeiros, A. I., et al. (2017). Soluble factors from biofilm of Candida albicans and Staphylococcus aureus promote cell death and inflammatory response. BMC Microbiol. 17:146. doi: 10.1186/s12866-0171031-5

Dominguez, E., Zarnowski, R., Sanchez, H., Covelli, A. S., Westler, W. M., Azadi, P., et al. (2018). Conservation and divergence in the Candida species biofilm matrix mannan-glucan complex structure, function, and genetic control. mBio 9:e00451-18.

Dominguez, E. G., Zarnowski, R., Choy, H. L., Zhao, M., Sanchez, H., Nett, J. E., et al. (2019). Conserved role for biofilm matrix polysaccharides in Candida auris drug resistance. $m$ Sphere 4:e00680-18.

Donlan, R. M. (2001a). Biofilms and device-associated infections. Emerg. Infect. Dis. 7, 277-281. doi: 10.3201/eid0702.010226

Donlan, R. M. (2001b). Biofilm formation: a clinically relevant microbiological process. Clin. Infect. Dis. 33, 1387-1392. doi: 10.1086/322972

Douglas, L. J. (2002). Medical importance of biofilms in Candida infections. Rev. Iberoam. Micol. 19, 139-143.
Douglas, L. J. (2003). Candida biofilms and their role in infection. Trends Microbiol. 11, 30-36. doi: 10.1016/s0966-842x(02)00002-1

Edwards, J. E. Jr., Lehrer, R. I., Stiehm, E. R., Fischer, T. J., and Young, L. S. (1978). Severe candidal infections: clinical perspective, immune defense mechanisms, and current concepts of therapy. Ann. Intern. Med. 89, 91-106.

Erwig, L. P., and Gow, N. A. (2016). Interactions of fungal pathogens with phagocytes. Nat. Rev. Microbiol. 14, 163-176. doi: 10.1038/nrmicro.2015.21

Fidel, P. L. Jr. (2002). Immunity to Candida. Oral Dis. 8(Suppl. 2), 69-75.

Harriott, M. M., Lilly, E. A., Rodriguez, T. E., Fidel, P. L., and Noverr, M. C. (2010). Candida albicans forms biofilms on the vaginal mucosa. Microbiology $156(\mathrm{Pt}$ 12), 3635-3644. doi: 10.1099/mic.0.039354-0

Hawser, S. P., Baillie, G. S., and Douglas, L. J. (1998). Production of extracellular matrix by Candida albicans biofilms. J. Med. Microbiol. 47, 253-256. doi: 10.1099/00222615-47-3-253

Hawser, S. P., and Douglas, L. J. (1994). Biofilm formation by Candida species on the surface of catheter materials in vitro. Infect. Immun. 62, 915-921. doi: 10.1128/iai.62.3.915-921.1994

Hoyer, A. R., Johnson, C. J., Hoyer, M. R., Kernien, J. F., and Nett, J. E. (2018). Echinocandin treatment of Candida albicans biofilms enhances neutrophil extracellular trap formation. Antimicrob. Agents Chemother. 62:e00797-18.

Jain, N., Kohli, R., Cook, E., Gialanella, P., Chang, T., and Fries, B. C. (2007). Biofilm formation by and antifungal susceptibility of Candida isolates from urine. Appl. Environ. Microbiol. 73, 1697-1703. doi: 10.1128/aem.02439-06

Johnson, C. C., Yu, A., Lee, H., Fidel, P. L. Jr., and Noverr, M. C. (2012). Development of a contemporary animal model of Candida albicans-associated denture stomatitis using a novel intraoral denture system. Infect. Immun. 80, 1736-1743. doi: 10.1128/iai.00019-12

Johnson, C. J., Cabezas-Olcoz, J., Kernien, J. F., Wang, S. X., Beebe, D. J., Huttenlocher, A., et al. (2016). The extracellular matrix of Candida albicans biofilms impairs formation of neutrophil extracellular traps. PLoS Pathog. 12:e1005884. doi: 10.1371/journal.ppat.1005884

Johnson, C. J., Kernien, J. F., Hoyer, A. R., and Nett, J. E. (2017). Mechanisms involved in the triggering of neutrophil extracellular traps (NETs) by Candida glabrata during planktonic and biofilm growth. Sci. Rep. 7:13065.

Kalan, L., and Grice, E. A. (2018). Fungi in the wound microbiome. Adv. Wound Care (New Rochelle) 7, 247-255. doi: 10.1089/wound.2017.0756

Kalan, L., Loesche, M., Hodkinson, B. P., Heilmann, K., Ruthel, G., Gardner, S. E., et al. (2016). Redefining the chronic-wound microbiome: fungal communities are prevalent, dynamic, and associated with delayed healing. mBio 7:e01058-16.

Katragkou, A., Chatzimoschou, A., Simitsopoulou, M., Georgiadou, E., and Roilides, E. (2011a). Additive antifungal activity of anidulafungin and human neutrophils against Candida parapsilosis biofilms. J. Antimicrob. Chemother. 66, 588-591. doi: 10.1093/jac/dkq466

Katragkou, A., Kruhlak, M. J., Simitsopoulou, M., Chatzimoschou, A., Taparkou, A., Cotten, C. J., et al. (2010). Interactions between human phagocytes and Candida albicans biofilms alone and in combination with antifungal agents. J. Infect. Dis. 201, 1941-1949.

Katragkou, A., Simitsopoulou, M., Chatzimoschou, A., Georgiadou, E., Walsh, T. J., and Roilides, E. (2011b). Effects of interferon-gamma and granulocyte colony-stimulating factor on antifungal activity of human polymorphonuclear neutrophils against Candida albicans grown as biofilms or planktonic cells. Cytokine 55, 330-334. doi: 10.1016/j.cyto.2011. 05.007

Kernien, J. F., Johnson, C. J., and Nett, J. E. (2017). Conserved Inhibition of neutrophil extracellular trap release by clinical Candida albicans biofilms. J. Fungi 3:49. doi: 10.3390/jof3030049

Kojic, E. M., and Darouiche, R. O. (2004). Candida infections of medical devices. Clin. Microbiol. Rev. 17, 255-267. doi: 10.1128/cmr.17.2.255-267.2004

Kucharikova, S., Neirinck, B., Sharma, N., Vleugels, J., Lagrou, K., and Van Dijck, P. (2015). In vivo Candida glabrata biofilm development on foreign bodies in a rat subcutaneous model. J. Antimicrob. Chemother. 70, 846-856. doi: $10.1093 / \mathrm{jac} / \mathrm{dku} 447$

Kuhn, D. M., Chandra, J., Mukherjee, P. K., and Ghannoum, M. A. (2002). Comparison of biofilms formed by Candida albicans and Candida parapsilosis on bioprosthetic surfaces. Infect. Immun. 70, 878-888.

Kumamoto, C. A., and Vinces, M. D. (2005). Alternative Candida albicans lifestyles: growth on surfaces. Annu. Rev. Microbiol. 59, 113-133. 
Lazzell, A. L., Chaturvedi, A. K., Pierce, C. G., Prasad, D., Uppuluri, P., and Lopez-Ribot, J. L. (2009). Treatment and prevention of Candida albicans biofilms with caspofungin in a novel central venous catheter murine model of candidiasis. J. Antimicrob. Chemother. 64, 567-570. doi: 10.1093/jac/ dkp242

Lee, H., Yu, A., Johnson, C. C., Lilly, E. A., Noverr, M. C., and Fidel, P. L. Jr. (2011). Fabrication of a multi-applicable removable intraoral denture system for rodent research. J. Oral Rehabil. 38, 686-690. doi: 10.1111/j.1365-2842.2011. 02206.x

Lewis, R. E., Kontoyiannis, D. P., Darouiche, R. O., Raad, I. I., and Prince, R. A. (2002). Antifungal activity of amphotericin B, fluconazole, and voriconazole in an in vitro model of Candida catheter-related bloodstream infection. Antimicrob. Agents Chemother. 46, 3499-3505. doi: 10.1128/aac.46.11.34993505.2002

Magill, S. S., Edwards, J. R., Bamberg, W., Beldavs, Z. G., Dumyati, G., Kainer, M. A., et al. (2014). Multistate point-prevalence survey of health careassociated infections. N. Engl. J. Med. 370, 1198-1208. doi: 10.1056/nejmoa13 06801

Mitchell, K. F., Zarnowski, R., Sanchez, H., Edward, J. A., Reinicke, E. L., Nett, J. E., et al. (2015). Community participation in biofilm matrix assembly and function. Proc. Natl. Acad. Sci. U.S.A. 112, 4092-4097. doi: 10.1073/pnas.142143 7112

Nash, E. E., Peters, B. M., Lilly, E. A., Noverr, M. C., and Fidel, P. L. Jr. (2016). A murine model of Candida glabrata vaginitis shows no evidence of an inflammatory immunopathogenic response. PLoS One 11:e0147969. doi: 10. 1371/journal.pone.0147969

Nett, J. E., Brooks, E. G., Cabezas-Olcoz, J., Sanchez, H., Zarnowski, R., Marchillo, K., et al. (2014). Rat indwelling urinary catheter model of Candida albicans biofilm infection. Infect. Immun. 82, 4931-4940. doi: 10.1128/iai.02284-14

Nett, J. E., Marchillo, K., Spiegel, C. A., and Andes, D. R. (2010). Development and validation of an in vivo Candida albicans biofilm denture model. Infect. Immun. 78, 3650-3659. doi: 10.1128/iai.00480-10

Nett, J. E., Zarnowski, R., Cabezas-Olcoz, J., Brooks, E. G., Bernhardt, J., Marchillo, K., et al. (2015). Host contributions to construction of three device-associated Candida albicans biofilms. Infect. Immun. 83, 4630-4638. doi: 10.1128/iai. 00931-15

Nieminen, M. T., Hernandez, M., Novak-Frazer, L., Kuula, H., Ramage, G., Bowyer, P., et al. (2014). DL-2-hydroxyisocaproic acid attenuates inflammatory responses in a murine Candida albicans biofilm model. Clin. Vaccine Immunol. 21, 1240-1245. doi: 10.1128/cvi.00339-14

Nobile, C. J., and Johnson, A. D. (2015). Candida albicans biofilms and human disease. Annu. Rev. Microbiol. 69, 71-92.

Pathirana, R. U., McCall, A. D., Norris, H. L., and Edgerton, M. (2019). Filamentous non-albicans Candida species adhere to Candida albicans and benefit from dual biofilm growth. Front. Microbiol. 10:1188. doi: 10.3389/fmicb.2019. 01188

Peters, B. M., Jabra-Rizk, M. A., Scheper, M. A., Leid, J. G., Costerton, J. W., and Shirtliff, M. E. (2010). Microbial interactions and differential protein expression in Staphylococcus aureus -Candida albicans dual-species biofilms. FEMS Immunol. Med. Microbiol. 59, 493-503. doi: 10.1111/j.1574-695x.2010. 00710.x

Pfaller, M. A., and Diekema, D. J. (2007). Epidemiology of invasive candidiasis: a persistent public health problem. Clin. Microbiol. Rev. 20, 133-163. doi: 10.1128/cmr.00029-06

Ramage, G., Vandewalle, K., Wickes, B. L., and Lopez-Ribot, J. L. (2001). Characteristics of biofilm formation by Candida albicans. Rev. Iberoam. Micol. $18,163-170$.

Redding, S. W. (2001). The role of yeasts other than Candida albicans in oropharyngeal candidiasis. Curr. Opin. Infect. Dis. 14, 673-677. doi: 10.1097/ 00001432-200112000-00002

Ricicova, M., Kucharikova, S., Tournu, H., Hendrix, J., Bujdakova, H., Van Eldere, J., et al. (2010). Candida albicans biofilm formation in a new in vivo rat model. Microbiology 156(Pt 3), 909-919. doi: 10.1099/mic.0.033530-0

Schinabeck, M. K., Long, L. A., Hossain, M. A., Chandra, J., Mukherjee, P. K., Mohamed, S., et al. (2004). Rabbit model of Candida albicans biofilm infection: liposomal amphotericin B antifungal lock therapy. Antimicrob. Agents Chemother. 48, 1727-1732. doi: 10.1128/aac.48.5.1727-1732.2004
Sellam, A., Al-Niemi, T., McInnerney, K., Brumfield, S., Nantel, A., and Suci, P. A. (2009). A Candida albicans early stage biofilm detachment event in rich medium. BMC Microbiol. 9:25. doi: 10.1186/1471-2180-9-25

Sherry, L., Ramage, G., Kean, R., Borman, A., Johnson, E. M., Richardson, M. D., et al. (2017). Biofilm-forming capability of highly virulent, multidrug-resistant Candida auris. Emerg. Infect. Dis. 23, 328-331. doi: 10.3201/eid2302.161320

Simitsopoulou, M., Chlichlia, K., Kyrpitzi, D., Walsh, T. J., and Roilides, E. (2018). Pharmacodynamic and immunomodulatory effects of micafungin on host responses against biofilms of Candida parapsilosis in comparison to those of Candida albicans. Antimicrob. Agents Chemother. 62:e00478-18.

Sultan, A. S., Rizk, A. M., Vila, T., Ji, Y., Masri, R., and Jabra-Rizk, M. A. (2019). Digital design of a universal rat intraoral device for therapeutic evaluation of a topical formulation against Candida-associated denture stomatitis. Infect. Immun. 87:e00617-19.

Tati, S., Davidow, P., McCall, A., Hwang-Wong, E., Rojas, I. G., Cormack, B., et al. (2016). Candida glabrata binding to Candida albicans hyphae enables its development in oropharyngeal candidiasis. PLoS Pathog. 12:e1005522. doi: 10.1371/journal.ppat.1005522

Tobouti, P. L., Casaroto, A. R., de Almeida, R. S., de Paula Ramos, S., Dionisio, T. J., Porto, V. C., et al. (2016). Expression of secreted aspartyl proteinases in an experimental model of Candida albicans-associated denture stomatitis. J. Prosthodont. 25, 127-134. doi: 10.1111/jopr.12285

Townsend, E. M., Sherry, L., Kean, R., Hansom, D., Mackay, W. G., Williams, C., et al. (2017). Implications of antimicrobial combinations in complex wound biofilms containing fungi. Antimicrob. Agents Chemother. 61:e00672-17.

Townsend, E. M., Sherry, L., Rajendran, R., Hansom, D., Butcher, J., Mackay, W. G., et al. (2016). Development and characterisation of a novel three-dimensional inter-kingdom wound biofilm model. Biofouling 32, 1259-1270. doi: 10.1080/ 08927014.2016.1252337

Tumbarello, M., Posteraro, B., Trecarichi, E. M., Fiori, B., Rossi, M., Porta, R., et al. (2007). Biofilm production by Candida species and inadequate antifungal therapy as predictors of mortality for patients with candidemia. J. Clin. Microbiol. 45, 1843-1850. doi: 10.1128/jcm.00131-07

Uppuluri, P., Acosta Zaldivar, M., Anderson, M. Z., Dunn, M. J., Berman, J., Lopez Ribot, J. L., et al. (2018). Candida albicans dispersed cells are developmentally distinct from biofilm and planktonic cells. mBio 9:e01338-18.

Uppuluri, P., Chaturvedi, A. K., Srinivasan, A., Banerjee, M., Ramasubramaniam, A. K., Kohler, J. R., et al. (2010). Dispersion as an important step in the Candida albicans biofilm developmental cycle. PLoS Pathog. 6:e1000828. doi: 10.1371/ journal.ppat.1000828

Urban, C. F., Reichard, U., Brinkmann, V., and Zychlinsky, A. (2006). Neutrophil extracellular traps capture and kill Candida albicans yeast and hyphal forms. Cell Microbiol. 8, 668-676. doi: 10.1111/j.1462-5822.2005.00659.x

Vipulanandan, G., Herrera, M., Wiederhold, N. P., Li, X., Mintz, J., Wickes, B. L., et al. (2018). Dynamics of mixed-Candida species biofilms in response to antifungals. J. Dent. Res. 97, 91-98. doi: 10.1177/0022034517729351

Wall, G., Montelongo-Jauregui, D., Vidal Bonifacio, B., Lopez-Ribot, J. L., and Uppuluri, P. (2019). Candida albicans biofilm growth and dispersal: contributions to pathogenesis. Curr. Opin. Microbiol. 52, 1-6. doi: 10.1016/j. mib.2019.04.001

Wang, X., and Fries, B. C. (2011). A murine. J. Med. Microbiol. 60(Pt 10), 1523-1529.

Wisplinghoff, H., Bischoff, T., Tallent, S. M., Seifert, H., Wenzel, R. P., and Edmond, M. B. (2004). Nosocomial bloodstream infections in US hospitals: analysis of 24,179 cases from a prospective nationwide surveillance study. Clin. Infect. Dis. 39, 309-317. doi: 10.1086/421946

Xie, Z., Thompson, A., Sobue, T., Kashleva, H., Xu, H., Vasilakos, J., et al. (2012). Candida albicans biofilms do not trigger reactive oxygen species and evade neutrophil killing. J. Infect. Dis. 206, 1936-1945. doi: 10.1093/infdis/jis607

Yano, J., Lilly, E., Barousse, M., and Fidel, P. L. Jr. (2010). Epithelial cellderived S100 calcium-binding proteins as key mediators in the hallmark acute neutrophil response during Candida vaginitis. Infect. Immun. 78, 5126-5137. doi: 10.1128/iai.00388-10

Yano, J., Noverr, M. C., and Fidel, P. L. (2012). Cytokines in the host response to Candida vaginitis: identifying a role for non-classical immune mediators, S100 alarmins. Cytokine 58, 118-128. doi: 10.1016/j.cyto.2011. 11.021 
Yano, J., Yu, A., Fidel, P. L. Jr., and Noverr, M. C. (2019). Candida glabrata has no enhancing role in the pathogenesis of Candida-associated denture stomatitis in a rat model. mSphere 4:e00191-19.

Zago, C. E., Silva, S., Sanita, P. V., Barbugli, P. A., Dias, C. M., Lordello, V. B., et al. (2015). Dynamics of biofilm formation and the interaction between Candida albicans and methicillin-susceptible (MSSA) and -resistant Staphylococcus aureus (MRSA). PLoS One 10:e0123206. doi: 10.1371/journal.pone.012 3206

Zarnowski, R., Westler, W. M., Lacmbouh, G. A., Marita, J. M., Bothe, J. R., Bernhardt, J., et al. (2014). Novel entries in a fungal biofilm matrix encyclopedia. mBio 5:e01333-14.

Zawrotniak, M., Bochenska, O., Karkowska-Kuleta, J., Seweryn-Ozog, K., Aoki, W., Ueda, M., et al. (2017). Aspartic proteases and major cell wall components in Candida albicans trigger the release of neutrophil extracellular traps. Front. Cell. Infect. Microbiol. 7:414. doi: 10.3389/fcimb.2017. 00414

Conflict of Interest: The authors declare that the research was conducted in the absence of any commercial or financial relationships that could be construed as a potential conflict of interest.

Copyright (c) 2020 Eix and Nett. This is an open-access article distributed under the terms of the Creative Commons Attribution License (CC BY). The use, distribution or reproduction in other forums is permitted, provided the original author(s) and the copyright owner(s) are credited and that the original publication in this journal is cited, in accordance with accepted academic practice. No use, distribution or reproduction is permitted which does not comply with these terms. 